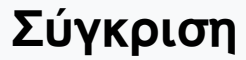

Tóp. 11 (2000)

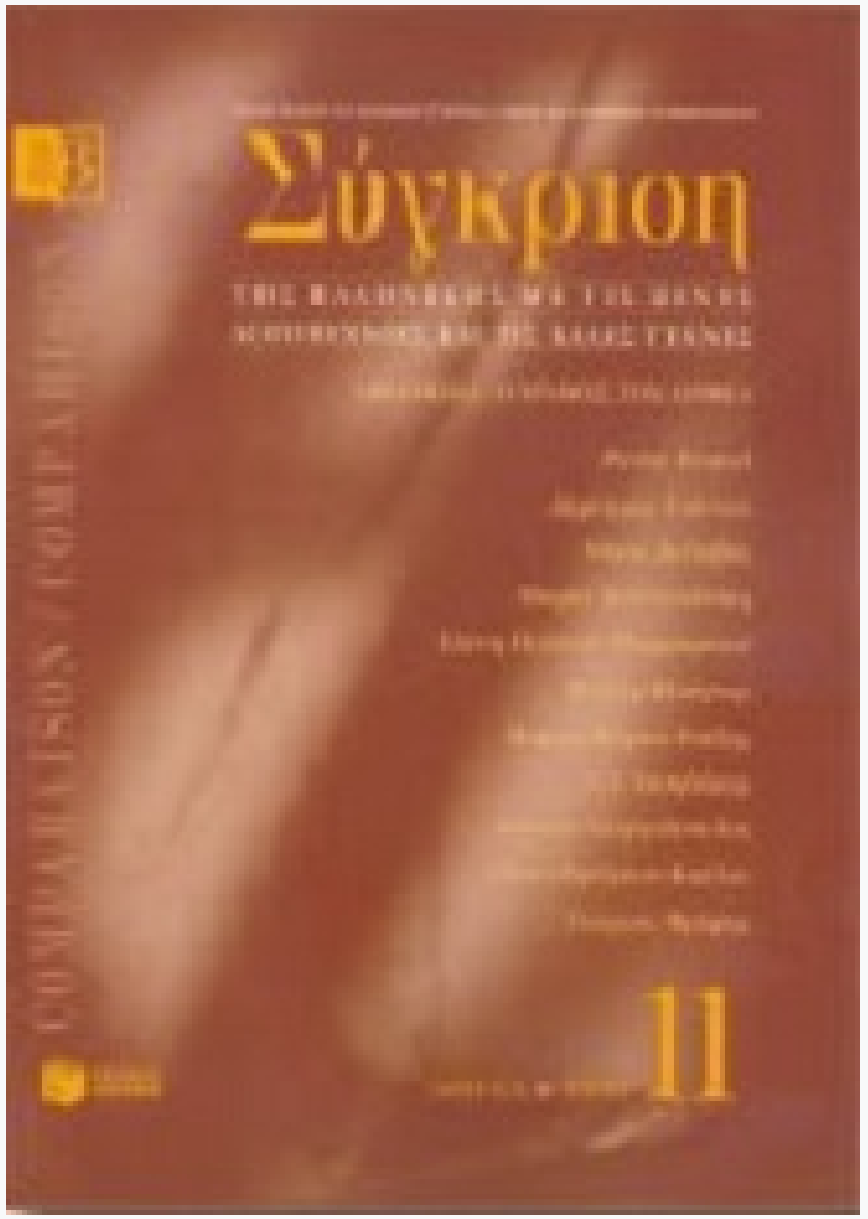

\section{Orphée Moderne}

\section{Pierre Brunel}

doi: $10.12681 /$ comparison.10761

\section{Copyright $\odot$ 2016, Pierre Brunel}

\section{@ $\odot \Theta \odot$}

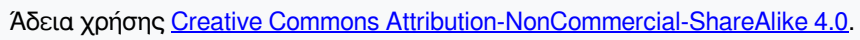

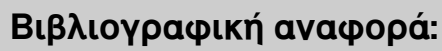

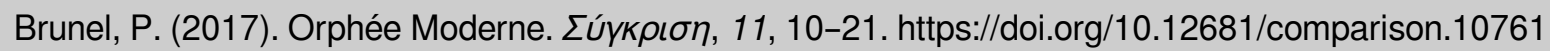




\section{P I E R R E B R U N E L}

\section{Orphée Moderne}

Je souhaite d'abord remercier la Société grecque de littérature comparée, son président, le professeur Costas Sterghiopoulos, son vice-président, le professeur Siaflekis, et l'université d'Athènes pour leur invitation. Je suis honoré et heureux de participer à ce beau colloque, mais il y a pour moi une raison supplémentaire de satisfaction. J'ai imaginé en effet l'an dernier d'organiser tout un cycle de manifestations littéraires autour du mythe d'Orphée, qui me fascine depuis longtemps, et je tenais beaucoup à ce que ce cycle fût inauguré à Athènes. Merci d'avoir entendu mon appel, et de m'avoir donné ample satisfaction. Le cycle devrait s'achever en novembre par une conférence que je donnerai à Bruxelles. Ce lieu aussi est symbolique. Il peut paraître inutile de rappeler tout d'abord qu'Orphée est grec. Il faut bien suivre l'évolution du mythe et voir que de mythe grec, il est devenu européen. On pense à Hugo von Hofmannsthal parlant du mythe de notre existence européenne («der Mythos unseres europäischen Daseins»), qui est aussi «création de notre monde intellectuel» affirmant sans cesse «le cosmos contre le chaos».

Une pareille extension dans l'espace va de pair avec une remarquable continuité dans le temps. Et de même qưil peut paraître audacieux de passer d'un Orphée grec à un Orphée européen, il est à la fois bouleversant et satisfaisant de passer d'un Orphée archaïque à un Orphée moderne. Je dirai même que l'adjectif jure, dans ce dernier cas, avec le substantif, et qu' «Orphée moderne», titre que j'ai choisi pour cette conférence, a des allures d'oxymore. Mais le fait que cet oxymore corresponde à une réalité littéraire et artistique suffit à justifier un semblable sujet et à autoriser une réflexion, qui restera ici modeste, sur cette alliance de mots.

Je me demanderai d'abord, sans vouloir anticiper sur la conférence du professeur Fanis Kakridis sur «Orphée: le noyau ancien», quel est et qui est l'Orphée archaïque. Il faudrait se tourner ici vers l'étude la plus savante, la plus solide, qui ait jamais été faite sur ce sujet. Alors au début d'une brillante carrière, Jacques Heurgon, qui fut l'un des grands maîtres des études latines à la Sorbonne, et qui a été mon professeur, disparu depuis peu de temps, donnait en 1932, dans le tome XLIX des Mélanges d'archéologie et d'histoire de l'École Française de Rome, un article substantiel (p. 5 à 60) intitulé «Orphée et Eurydice avant Virgile». Il ne 
minimisait pas la place exceptionnelle d'Orphée dans le Livre IV des Géorgiques de Virgile, au ${ }^{\text {er }}$ siècle avant Jésus-Christ, et il savait bien le rôle fondateur de ce texte, moins pour l'Orphée au miel dont a parlé Marcel Détienne, que pour la mise en place de l'histoire tragique des amours d'Orphée et Eurydice, traversées par la mort, traversant la mort et pourtant finissant par s'y engloutir. Mais il permettait de découvrir que l'apparente «révolution virgilienne» pourrait bien n'être qu'une illusion. Virgile n'invente jamais, d'une manière générale. Il conduit à la perfection les éléments d'un matériau emprunté. Dans ce cas précis, «il ne s'est écarté $\mathrm{du}$ thème en quelque sorte officiel que parce qu'une forme, moins connue, de la légende, l'avait intéressé et enhardi». Déjà Phèdre, le jeune disciple de Socrate, dans Le Banquet de Platon, faisait allusion, en la réprouvant, à une descente d'Orphée dans l'Hadès à la recherche de son épouse perdue: une pareille catabase n'était pas possible sans franchissement d'une limite sacrée, sans le viol d'un interdit majeur - pour un vivant, entrer chez les morts.

Ce document écrit est à rapprocher d'un document archéologique capital, le bas relief «Orphée, Eurydice, Hermès», contemporain de Phidias, donc du $\mathrm{V}^{e}$ siècle avant Jésus-Christ, dont nous ne connaissons que trois copies romaines. La descente d'Orphée dans l'Hadès n'est pas attestée avant cette époque, où se sont constitués les plus anciens monuments de la littérature orphique, dans le cadre d'une vaste entreprise de refonte et de codification des traditions religieuses dont, à Athènes, les fils de Pisistrate se sont faits les patrons. La catabase a bientôt pris un aspect plus profane que mystique, elle est devenue une histoire d'amour touchante, un modèle auquel songe Admète dans l'Alceste d'Euripide (438 av. J.- C.), ou prétexte à une généralisation abusive («Orphée ramenant les morts de l'Hadès») dans un discours d'apparat d'Isocrate, Bousiris (vers 390 av. J. - C.). L 'épouse d'Orphée a été d'abord désignée sous le nom d'Argiopè, ou Agriopè, et elle se trouve désignée pour la première fois (dans l'état des documents écrits dont nous disposons) dans le Chant funèbre en l'honneur de son maître Bion de Moschos, ou attribué à Moschos, à l'époque alexandrine. Le texte est remarquable, puisque le nom de Corè, épouse d'Hadès et reine des Enfers, y figure à côté de celui d'Eurydice:

«Ton chant ne sera pas sans récompense; comme jadis à Orphée, pour prix des doux sons de sa lyre, Corè accorda le retour d'Eurydice, elle te renverra, Bion, à tes montagnes».

Je tiens déjà là quelques éléments qui permettent de passer de l'Orphée archaïque à l'Orphée moderne, si je cherche à retrouver cet Orphée premier à la faveur de textes modernes. 
1. La quête d'un Orphée archaïque

Curieusement, Orphée est presque absent de la grande interrogation de Nietzsche sur les origines de la musique. À peine est-il nommé dans le chapitre 19 de La Naissance de la tragédie (1872). On a l'impression que le philosophe allemand - ou, tout aussi bien, le philologue bâlois - a été gêné par ce qu'est devenu Orphée au moment de la Renaissance et dans la mythologie «naturaliste», en particulier la mythologie solaire de Max Müller. Mallarmé, tenant de ce mouvement de pensée qui cherche à retrouver dans les grands mythes des archétypes naturels (lever du soleil, coucher du soleil, par exemple), a dévoilé dans Les Dieux antiques, un manuel de mythologie publié en 1880 et largement tributaire d'un ouvrage similaire de l'Anglais George William Cox, un Orphée éolien, une figure du vent, tandis qu'Eurydice correspondrait au «vaste jaillissement de l'aurore dans le ciel» avant qu'elle ne soit mordue par le «serpent des ténèbres». Ou encore, «le pèlerinage d'Orphée [...] représente le voyage que, pendant les heures de la nuit, le Soleil passait pour accomplir afin de ramener, au matin, l'Aurore, dont il cause la disparition par sa splendeur éblouissante $»^{1}$. C'est dans cette mouvance qu'il faut sans doute situer l'Hymne orphique (1904) de Costis Palamas:

«De nuit je me mets en route,

de nuit je monte au sommet inacessible.

Que je sois seul, que je sois le premier à saluer la lumière apollinienne, lorsqu'en bas chez les hommes

régneront encore le sommeil et les ténèbres».

(trad. de Georges Archimandritis)

Il est un autre texte remarquable, et rarement cité, de Mallarmé, qui mérite d'être pris en compte à ce sujet. Il s'agit d'un hommage au poète parnassien Théodore de Banville, en 1892, à l'occasion de l'inauguration d'un monument au Jardin du Luxembourg. Après la mort de Banville, «nul mieux ne représente maintenant le Poëte, l'invincible, classique Poëte soumis à la déesse et vivant parmi le charme oublié des héros et des roses. Sa parole, sans fin, l'ambroisie, que seul tarit le cri ivre de toute gloire... Les vents qui parlent d'effarement et de la nuit, les abîmes pittoresques de la région, il ne les veut entendre ni ne doit les voir: il marche à travers l'enchantement édénéen, désignant à jamais la noblesse des rayons et l'éclatante blancheur du lys enfant - la terre heureuse! Ainsi dut être qui le premier reçut des dieux la voix et dit l'ode éblouie avant notre aïeul Orphée $»^{2}$. Non seulement, on le voit, Mallarmé cherche à retrouver l'Orphée archaïque, l'«aïeul Orphée», mais il imagine un Orphée avant Orphée, un aïeul avant l'aïeul, celui qui reçut des dieux, ou du dieu, le don de la musique. 


\section{Orphée, Eurydice, Hermès}

Le document archéologique principal auquel, après Jacques Heurgon, j'ai fait allusion tout à l'heure, le bas-relief «Orphée, Eurydice, Hermès», est à l'origine d'un des plus beaux textes du XX $\mathrm{XX}^{\mathrm{e}}$ siècle consacrés au mythe d'Orphée. La présence d'Hermès se justifie, puisqu'il est traditionnellement le dieu psychopompe, celui qui conduit les âmes des morts, soit vers l'Hadès (c'est le cas dans le chant XXIV de l'Odyssée, quand il entraîne derrière lui les âmes des prétendants tués par Ulysse à son retour en Ithaque), soit de l'Hadès vers le monde des vivants (puisque tel est le trajet inverse auquel Eurydice semble avoir été autorisée par les dieux des Enfers eux-mêmes).

Dans l'un de ses Neue Gedichte (1907), Rainer-Maria Rilke s'est inspiré de ce bas - relief, ou du moins d'une copie, peut-être de provenance éleusinienne, conservée au Musée de Naples, et présentant le trio. Le titre du poème, «Orpheus. Eurydike. Hermes», sépare les noms seulement par un point, comme si chacun d'entre eux était enfermé dans sa solitude essentielle (on sait l'importance de ce thème chez Rilke). Nous sommes sur le chemin du retour, et même si Orphée ne regarde pas derrière lui, comme cela lui a été interdit, il est à l'écoute de tout ce qui peut se passer derrière lui. Est-il si nécessaire d'ailleurs qu'Eurydice suive son époux, l'accompagne sur terre, reprenne à ses côtés la vie d'une femme, qu'elle retrouve la lumière du soleil? Non, car pour Rilke, qui fait ici subir au sens du mythe une mutation importante, elle est comblée par la mort:

«Sie war in sich. Und ihr Gestorbensein erfüllte sie wie Fülle.

Wie eine Frucht von Sussigkeit und Dunkel, so war sie voll von ihrem grossen Tode, der also neu war, dass sie nichts begriff.» («Elle était en elle-même. Et sa mort la remplissait comme une abondance. Comme un fruit de douceur et de ténèbres, elle était pleine de sa mort énorme et neuve et ne comprenait rien»). ${ }^{3}$

\section{Eurydice et Corè}

Pour bien comprendre l'histoire d'Eurydice et d'Orphée, il faut voir qu'elle vient redoubler l'histoire de Corè et d'Hadès. En effet, le dieu des Enfers a enlevé la fille de Déméter alors qu'elle cueillait des fleurs dans la prairie, de la même manière que, par l'intermédiaire du serpent, il a arraché Eurydice à la vie. Et il accède à la prière d'Orphée comme il a dû faire une concession à Déméter, en acceptant qu'elle revienne auprès 
d'elle la moitié du temps, en lui réservant l'autre moitié. Ce parallèle nest nullement un fait de hasard. Il confirme la structure mythique de la réduplication (le «conte-redire» auquel Claude Lévi-Strauss a accordé une place majeure à côté du «contredire»), il laisse deviner que, dans l'antique religion grecque, et surtout sans doute dans les cultes à mystères, un lien existait entre les deux mythes et contribuait à l'épanouissement d'une mystique. C'est dans le Livre $\mathrm{X}$ des Métamorphoses d'Ovide, au $\mathrm{I}^{\text {er }}$ siècle après Jésus-Christ, qu'on trouve l'habile discours d'Orphée au couple royal des Enfers (ici Pluton et Proserpine) où il met en parallèle les deux rapts. Au XIX siècle, Alfred Tennyson l'a largement exploité dans son grand poème «Demeter and Persephone», et au XXe siècle nul n'y est sans doute plus sensible, même si les allusions restent discrètes, qu'Yves Bonnefoy, dans son troisième recueil, Pierre écrite (1965), et plus particulièrement dans la deuxième partie du «Dialogue d'angoisse et de désir»:

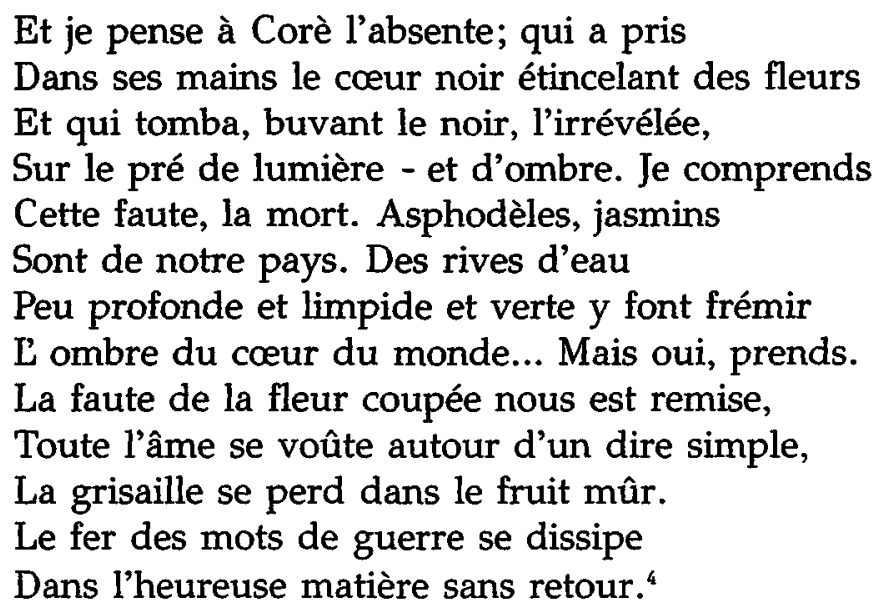

Eurydice, il est vrai, nest pas nommée. Mais son ombre rôde dans la poésie d'Yves Bonnefoy, depuis Du mouvement et de l'immobilité de Douve, le recueil qui l'a imposé en 1953. La grande cantatrice anglaise Kathleen Ferrier, prématurément disparue en 1953, à qui est dédié le plus beau poème du second recueil, «Hier régnant désert», en 1958, serait plutôt un nouvel Orphée. Elle avait d'ailleurs chanté le rôle dans la version italienne de l'opéra de Gluck, et nous en avons conservé un enregistrement précaire. Elle «conna(ît) les deux rives» ${ }^{5}$, comme Orphée, comme Vera Ouckama Knoop, l'autre figure orphique féminine des Sonnets à Orphée de Rilke, en 1922.

On a glosé «fer», dans le vers 3 du poème «À la voix de Kathleen Ferrier», par la première syllabe du nom de la cantatrice. L'hypothèse est ingénieuse, un peu facile, trop sans doute quand on a affaire à un poète aussi exigeant que Bonnefoy. Le «fer» est traditionnellement l'emblème du 
héros guerrier, du héros épique, alors que la lyre est celui du héros poète, du héros lyrique. C'est Achille d'un côté, Orphée de l'autre. «Le fer des mots de guerre», dans le poème de Pierre écrite, vient confirmer cette hypothèse, et créer un lien entre le recueil de 1958 et celui de 1965. Il faut que le fer fasse silence pour que place soit faite à la musique d'Orphée. Il faut que la lumière du glaive se voile pour que «Là-bas, parmi ces roseaux gris dans la lumière, Il semble que tu puises de l'éternel».

$\mathrm{Si}$ on considère que c'est au XIX siècle que naît la modernité, associée au nom de Baudelaire (Le Peintre de la vie moderne), il faudrait pouvoir assister à la naissance d'Orphée moderne dans ce siècle. S'il reste un sujet académique, Orphée est pourtant renouvelé par les artistes romantiques (Delacroix, Liszt, Hugo) et à la fin du siècle (avec en particulier la peinture de Gustave Moreau). C'est de ce sujet que je traiterai au Conservatoire de musique de Lausanne, le 6 février 1998, dans ce qui sera ma deuxième intervention dans ce cycle Orphée. Il est clair qu’avec Mallarmé, à la fin du $\mathrm{XIX}^{e}$ siècle, il devient une figure emblématique de la parole et de la grande interrogation qu'elle suscite. Le texte essentiel est alors le célèbre sonnet dit en $-x$, celui qui commence par «Ses purs ongles très haut dédiant leur onyx». Il remonte à 1868 , mais il n’a été publié pour la première fois, et dans sa version définitive, qu'en 1887 dans les Poésies.

«Le Maître» qui est allé «puiser des pleurs au Styx», s'il n’est pas Orphée, est du moins celui qui recommence l'aventure d'Orphée. N'en ramènerait-il que le «ptyx», que «ce seul objet dont le Néant s'honore», il est une figure orphique du poète. Il n'est pas d'autre Eurydice perdue que la parole poétique. Et dès 1868, dans une lettre à Henri Cazalis qui lui avait en quelque sorte commandé le poème, Mallarmé le définissait comme le projet d'une «étude sur la parole». Il était naturel qu’à partir de là, la recherche de Mallarmé s'orientât vers «l'explication orphique de la terre». L' expression est souvent citée, mais on ignore généralement qu'elle est extraite d'un texte adressé le 16 novembre 1885 à Verlaine comme une lettre. On le connait sous le titre d' «Autobiographie» ${ }^{6}$. Cette explication orphique est inséparable du «Livre», et c'est pourquoi elle correspond au «seul devoir du poète et (au) jeu littéraire par excellence». Elle devait être le point d'aboutissement de la quête mallarméenne de la poésie des origines, de l'«aïeul Orphée».

$\mathrm{Au}$ début du XXe siècle, c'est sans doute Guillaume Apollinaire qui a contribué de la manière la plus complète et la plus remarquable à l'illustration et au renouvellement du mythe littéraire d'Orphée. J'ai déjà eu l'occasion de traiter ce sujet, au moins partiellement, dans mon livre Apollinaire entre deux mondes.-Mythocritique II, dont le chapitre III s'intitule précisément «Orphée moderne»? Je ne cherche pas à redoubler ici, mais je 
voudrais dégager quelques aspects essentiels d'une semblable reprise moderne du mythe ancien.

\section{Une reprise complète}

On réduit souvent le mythe d'Orphée à l'histoire d'Orphée et d'Eurydice. Mais même la version virgilienne ou la version ovidéenne représentent plus que cela. Si on essaie de constituer ce mythe en ensemble, on découvre trois axes essentiels, ceux que j'ai retenus pour ma présentation du mythe d'Orphée dans le Dictionnaire des mythes littéraires ${ }^{8}$, et qui serviront d'axes aussi au prochain colloque de Paris: Orphée magicien (avec en particulier son intervention dans la geste des Argonautes), Orphée et Eurydice, Orphée et les Bacchantes (la mort d'Orphée).

Orphée magicien est représenté par Le Bestiaire ou Cortège d'Orphée, qui prit naissance dans l'atelier de Picasso en 1906, fut publié dans une première version en 1908 dans le numéro 24 de la revue La Phalange sous le titre «La marchande des quatre saisons ou le bestiaire mondain», et aboutit à la version définitive de 1910, enrichie de bois de Raoul Dufy, avant que Francis Poulenc ne mette quelques-uns de ces brefs poèmes en musique dans Le Bestiaire en 1919.

Orphée passe dès l'origine pour être celui qui, au son magique de sa lyre ou de sa voix, attire à lui les animaux et dompte les plus fauves d'entre eux. Palamas le représente encore dans son Eurydice:

«(...) et le son tout-puissant de ta lyre,

dompteur de tous les êtres du monde, sauvages ou dressés» (trad. G. Archimandritis).

C'est le prétexte pour Apollinaire à l'évocation amusée, tendre ou ironique, de divers animaux qui sont censés faire partie de ce cortège comme ils pourraient peupler l'arche de Noé, de la tortue au buf, du plus lourd, l'éléphant, aux plus petits,

«Rotifères, cirons, insectes

Et microbes plus merveilleux

Que les sept merveilles du monde

Et le palais de Rosemonde! $\gg^{9}$

Le recueil est ponctué de quatre poèmes intitulés «Orphée», évoquant tour à tour Orphée maître des secrets dans le Pimandre attribué à Hermès Trismégiste, Orphée conducteur des animalcules (c'est le poème cité), Orphée-Jésus, Orphée aux prises avec les Sirènes et essayant de lutter avec elles par la magie de la musique. Ce dernier aspect se rattache à Orphée Argonaute, un élément du mythe qui a longuement retenu Apollinaire et qu'illustrent de manièré originale certains poèmes d'Alcools, en 1913, comme le mystérieux «Lul de Faltenin». 
5. Le regard d'Orphée

Le plus beau poème orphique d'Apollinaire est sans aucun doute, dans Alcools précisément (1913), celui qui s'intitule «Cors de chasse»:

«Notre histoire est noble et tragique

Comme le masque d'un tyran

Nul drame hasardeux ou magique

Aucun détail indifférent

Ne rend notre amour pathétique

Et Thomas de Quincey buvant

L' opium poison doux et chaste

À sa pauvre Anne allait rêvant

Passons passons puisque tout passe

Je me retournerai souvent

Les souvenirs sont cors de chasse

Dont meurt le bruit parmi le vent. $\gg^{10}$

Le poème a été publié pour la première fois dans la revue Les Soirées de Paris, en septembre 1912. Il reflète une manière de deuil après l'abandon par la compagne bien-aimée, le peintre Marie Laurencin. Mais le nouvel Orphée, sans avoir besoin de se donner ce nom, et sans lui donner le nom d'Eurydice, transgresse volontairement et lucidement l'interdit du regard en arrière: «Je me retournerai souvent». La mémoire est la seule manière de fidélité à l'infidèle, la seule manière de combattre cette infidélité même, le seul refuge contre l'usure du temps, contre l'alcool du temps, «cet alcool brûlant comme ta vie / Ta vie que tu bois comme une eau-de-vie $»^{11}$.

\section{La mort d'Orphée}

Un récit en prose d'Apollinaire, Le Poète assassiné, fournit une excellente illustration, véritablement moderne, du troisième grand mythème, la mise à mort d'Orphée par les Bacchantes de Thrace, dont le Livre XI des Métamorphoses d'Ovide donne la version en quelque sorte canonique. Après la disparition définitive d'Eurydice, Orphée est revenu sur terre. Il se détourne de l'amour de sa femme vers l'amour des jeunes garçons, mais les femmes thraces, dévotes de Dionysos, ne peuvent l'accepter, et elles déchirent son corps selon le rite dionysiaque du diasparagmos. La version proprement ovidéenne a été reprise par Luciano Berio dans son Orfeo II, d'après l'opéra de Monteverdi, en 1984, tandis que Jean Cocteau, dans son film de.1949, Orphée, a représenté une foule agressive. Nikos Engonopoulos, grand représentant du Surréalisme en Grèce, a procédé à une semblable évocation du diasparagmos dans son poème «Orphée», du recueil Dans la vallée des roseraies (1978).

Apollinaire a mis au point son Poète assassiné en 1913 et 1914, donc 
peu de temps après la publication d'Alcools, la publication définitive intervenant en 1916. Le groupe surréaliste en présentera une nouvelle édition en 1927, et considérera ce texte comme une œuvre-phare de la modernité. Orphée se nomme ici Croniamantal. Il est le fils d'un musicien ambulant et d'une femme vénale qui, après nombre de mésaventures, a réussi à se faire épouser par un riche baron. Devenu poète, il a pour maîtresse Tristouse Ballerinette, qui est aussi la maîtresse du vulgaire Paponat. Il part en voyage à travers l'Europe (belle illustration déjà, à la faveur d'une parodie, d'Orphée européen!), accomplit certains des voyages faits par Apollinaire lui-même, par exemple quand il suivait Annie Playden, l'inspiratrice de «La Chanson du mal-aimé», revient à Marseille au moment où une foule en délire y acclame le grand Horace Tograth, dont la science, dit-on, tenait du miracle. «Comme Orphée», écrit alors Apollinaire, «tous les poètes étaient près d'une malemort $\gg^{12}$. Croniamantal en effet va y être frappé à mort par les nouvelles Bacchantes tandis que Tristouse, du bout de son parapluie, lui crèvera œun il. Cela ne l'empêchera pas de s'écrier: «Je confesse mon amour pour Tristouse Ballerinette, la poésie divine qui console mon âme»:

«Les femmes s'écartèrent vite, et un homme qui balançait un grand couteau posé sur sa main ouverte le lança de telle façon qu'il vint se planter dans la bouche ouverte de Croniamantal. D'autres hommes firent de même. Les couteaux se fichèrent dans le ventre, la poitrine, et bientôt il n'y eut plus sur le sol qu'un cadavre hérissé comme une bogue de châtaigne marine $\gg^{13}$.

L image, parodique ici de celle de Notre-Dame des sept douleurs, se trouvait déjà dans Alcools, comme les sept douleurs y étaient représentées par les sept épées de la «Chanson du mal-aimé». Ainsi, dans la série des «Rhénanes», à la fin de la «Rhénane d'automne»:

$« \grave{A}$ nos pieds roulaient les châtaignes

Dont les bogues étaient

Comme le cœur blessé de la madone

Dont on doute si elle eut la peau

Couleur des châtaignes d'automne»..$^{14}$

Il était dans la logique du temps qu'on cherchât à moderniser ce vieux sujet, Orphée, en l'adaptant au goût du jour, pour ne pas dire en le mettant à la mode. C'est apparemment l'aspect le plus superficiel d'Orphée moderne, celui sur lequel on me permettra de métendre le moins.

Les procédés les plus courants sont:

1. la mise en place de décors modernes (un buffet de gare dans 
l'Eurydice de Jean Anouilh, où Eurydice elle-même meurt dans un accident d'autobus, un café, le café des poètes dans le film de Jean Cocteau, Orphée, le carnaval de Rio dans le film de Marcel Camus, Orfeu negro).

2. Les avatars de l'emblème majeur, celui de la lyre: devenue l'immense lyre de l'Orphée roi de Victor Segalen, elle peut tout aussi bien être une guitare dans la pièce de Tennessee Williams, Orpheus descending (La Descente d'Orphée).

3. Les amalgames: celui d'Orphée et du Christ est particulièrement sensible dans le recueil qui imposa Pierre Emmanuel en 1941, Tombeau d'Orphée.

4. L'intervention de personnages nouveaux: Heurtebise, chez Cocteau, M. Henri dans l'Eurydice de Jean Anouilh.

5. Lorientation vers un sens nouveau. Ainsi, dans le film de Cocteau, Orphée croit ne plus aimer Eurydice et être amoureux de la Mort, superbement incarnée par la grande actrice Maria Casarès. Aussi effectue-t-il des voyages à travers le miroir (le souvenir de Lewis Carroll passe...) essentiellement pour rejoindre la princesse noire. Et quand Heurtebise lui demande: «Est-ce la mort que vous désirez rejoindre, ou Eurydice?», il répond, un peu honteux: «Les deux» $»^{15}$. Cette fascination s'explique dans la mesure où la princesse rend le poète à sa vocation et lui fait connaître de nouvelles vérités poétiques.

Orphée est d'autant plus séduit qu'il attendait un choc semblable, qu'il espérait une rencontre susceptible de l'écarter d'une réalité creuse, de l'éloigner de l'ordinaire qui constituait son existence avec Eurydice. Le poète part à la conquête de son œuvre, et il trouve la mort. La Mort part à la conquête du monde terrestre, et elle trouve l'amour. C'est elle, curieusement, qui pratique le regard en arrière. Elle se retourne vers son passé, et elle retrouve ainsi son ancienne humanité: sous ses vêtements stricts et austères se cache un cœur qui bat d'amour pour un vivant, et les traits de son visage ne parviennent plus à la réduire à la froideur calculatrice qu'elle montrait au début du film. Elle finit par représenter l'amour sublimé, l'amour le plus pur, celui qui ne comporte aucun mensonge, un amour si rédempteur que, précisément, il ne peut s'accomplir que dans le cheminement vers la mort, vers le grand dénouement tragique, mais cette fois accepté: la Mort meurt d'aimer, elle se sacrifie au poète pour lui offrir l'immortalité et le rendre à la vie.

Le drame de la princesse vient de ce qu'elle change les règles du jeu sans en avertir l'au-delà. Elle' agit sans avoir reçu d'ordre, et au contraire en transgressant les interdits de la mort. Elle détourne sa mission de son but initial. Elle décide de se replonger dans une humanité qui lui était 
interdite par son statut. «Je n'ai le droit d'aimer personne, et j'aime», ditelle elle-même, exposant lucidement le paradoxe qu'elle constitue ${ }^{16}$.

Ainsi ne fait-elle qu'approfondir l'oxymore orphique: non pas Orphée moderne, mais Orphée mort-vivant, celui que présentait sobrement mais fortement Gérard de Nerval dans le premier sonnet des Chimères, «El Desdichado»:

«Et j’ai deux fois vainqueur traversé l'Achéron».

Le mythe réussit ainsi cette impossible alliance des contraires, la vie et la mort, du moins temporairement, le temps d'une saison en enfer qui prélude à la mort de deux êtres qui resteront peut-être enfermés chacun dans sa solitude essentielle. À l'époque moderne, Maurice Blanchot, plus et mieux que tout autre, s'est enchanté de ces paradoxes d'Orphée et de ces oxymores orphiques, tant dans $L$ 'Espace littéraire (1955) que dans $L ' E n t r e t i e n$ infini (1969). Il a vu dans le désir «la séparation qui se fait elle-même attirante», «l'intervalle qui devient sensible», «l'absence qui retourne à la présence», la nuit qui devient jour, comme celle qu’a dû traverser Orphée pour rejoindre Eurydice aux Enfers.

Blanchot a lu aussi dans le mythe d'Orphée la nécessité et l'impossibilité de l'œuvre. Par l'épisode de la catabase surtout, il prête à un symbolisme riche de sens. La nuit représente l'espace littéraire, l'espace de l'œuvre, dans lequel l'artiste Orphée doit s'enfoncer pour saisir l'essence de l'œuvre qu'il ramènera au jour. Eurydice est la source et l'essence de l'œuvre, qu'Orphée saisit et perd en même temps. Orphée, par son regard, incarne l'inspiration, qui annonce l'œuvre et en même temps la voue à l'échec.

Sans doute un tel Orphée est-il excessivement intellectualisé. Mieux vaut revenir au mythe pris dans son ensemble, avec toutes ses séquences, comme a essayé de le faire Renaud Gagneux dans son opéra récent, Orphée. Ou mieux vaut encore faire d'un poète-mage trop figé dans son habit et dans son rôle, un éternel adolescent, tel l'Orphée bohémien d'Arthur Rimbaud dans «Ma Bohême», à l'automne de 1870, qui

«(...) rimant au milieu des ombres fantastiques,

Comme des lyres, (...) tir(ait) les élastiques

De (s)es souliers blessés, un pied près de (s)on cœur». 


\section{$\Sigma \eta \mu \varepsilon \iota \omega ́ \sigma \varepsilon \iota \varsigma$}

${ }^{1}$ Les Dieux antiques, repris dans Euvres complètes de Mallarmé, éd. de G. Jean-Aubry et Henri Mondor, Gallimard, Bibliothèque de la Pléiade, 1945, p. 1239-1240.

${ }^{2}$ Euvres complètes, éd. cit., p. 520521.

${ }^{3}$ Neue Gedichte, première partie, 1907, rééd. Insel Verlag, 1974, Insel Taschenburg, p. 67-71. Traduction française de Lorand Gaspar, dans Rainer-Maria Rilke, Euvres II, Poésie, éd. du Seuil, 1972, p. 215.

${ }^{4}$ Yves Bonnefoy, Poèmes, Mercure de France, 1978, p. 220.

${ }^{5}$ «À la voix de Kathleen Ferrier», ibid., p. 137.

${ }^{6}$ Euvres complètes de Mallarmé, éd. cit., p. 663.

7 Presses Universitaires de France, col. PUF/écriture, 1997, p. 63-82.

${ }^{8}$ Ed. du Rocher, 1988, deuxième éd., 1994, p. 1129-1139.
${ }^{9}$ Euvres poétiques d'Apollinaire, éd. de Marcel Adéma et Michel Décaudin, Gallimard, Bibliothèque de la Pléiade, 1965, p. 15.

${ }^{10}$ Ibid., p. 148.

${ }^{11}$ «Zone», premier poème d'Alcools, éd. cit., p. 44, en réalité le dernier dans l'ordre de la composition, donc postérieur lui aussi à la rupture avec Marie Laurencin.

${ }^{12}$ Euvres en prose d'Apollinaire, éd. de Michel Décaudin, tome I, Gallimard, Bibliothèque de la Pléiade, 1977, p. 294.

${ }^{13}$ Ibid., p. 299-300.

${ }^{14}$ Euvres poétiques d'Apollinaire, éd. cit., p. 120.

${ }^{15}$ Orphée, scénario du film, J'ai lu, 1987, p. 78.

${ }^{16}$ Ibid., p. 89.

\section{$\Pi \varepsilon \rho i \lambda \eta \psi \eta$}

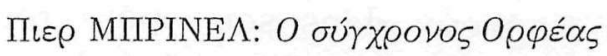

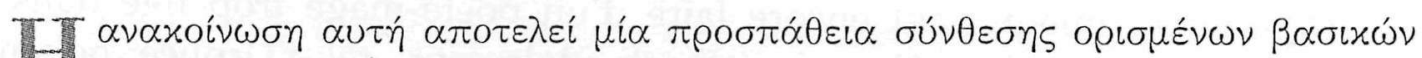

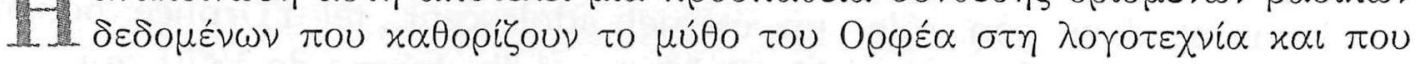

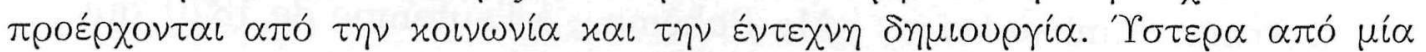

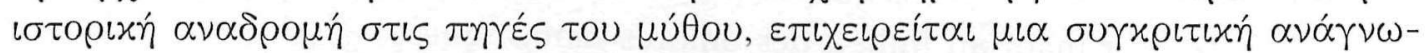

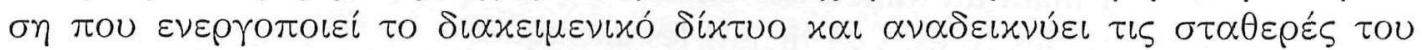

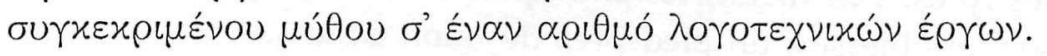

